\title{
"Hacker" et collaborer : dispositifs pour la formation de médiateurs culturels des sciences et techniques.
}

Loïc Petitgirard

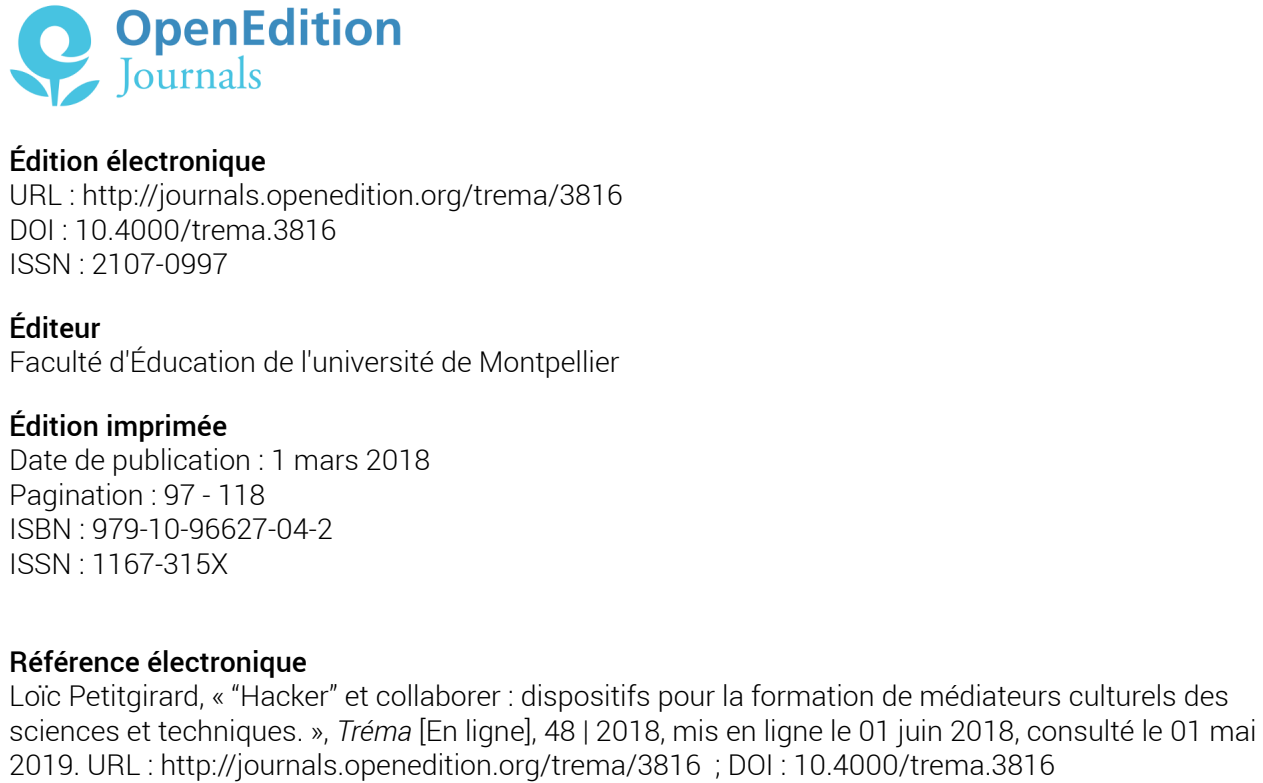

Édition électronique

URL : http://journals.openedition.org/trema/3816

DOI : 10.4000/trema.3816

ISSN : 2107-0997

Éditeur

Faculté d'Éducation de l'université de Montpellier

Édition imprimée

Date de publication : 1 mars 2018

Pagination : $97-118$

ISBN : 979-10-96627-04-2

ISSN : 1167-315X

Référence électronique

Loïc Petitgirard, «"Hacker" et collaborer : dispositifs pour la formation de médiateurs culturels des sciences et techniques. », Tréma [En ligne], 48 | 2018, mis en ligne le 01 juin 2018, consulté le 01 mai 2019. URL : http://journals.openedition.org/trema/3816 ; DOI : 10.4000/trema.3816

Ce document a été généré automatiquement le 1 mai 2019.

Trema 


\title{
"Hacker" et collaborer : dispositifs pour la formation de médiateurs culturels des sciences et techniques.
}

\author{
Loïc Petitgirard
}

\section{Introduction}

1 Cet article vise à problématiser un processus pédagogique proposé dans le cadre des formations à la médiation des sciences et des techniques, au Conservatoire national des arts et métiers (CNAM) à Paris. Au cours de son histoire le CNAM a endossé plusieurs rôles de «médiateur » entre sciences, techniques et société. C'est en lui-même un lieu où s'opère une médiation, de par l'existence en son cœur du "Musée des arts et métiers » (autrefois « Musée national des techniques », ou plus simplement " Musée du CNAM ») : le CNAM, et son Musée, déploient une mission de "culture scientifique et technique", étendue à tout le territoire national. Le CNAM est aussi un lieu de recherche et de formation sur ces questions d'interface entre sciences, techniques et société, qui a été porté par exemple par le Centre «Science, technique, société », piloté par Jean-Jacques Salomon de 1978 jusqu'en 1998. Les formations se sont renouvelées au fil de son histoire et elles s'inscrivent depuis 10 ans dans des diplômes, en formation continue pour adultes, et en adéquation avec la montée en professionnalisation des acteurs de la médiation ${ }^{1}$. Dans une institution à l'écoute des transformations des pratiques de médiation, nous avons pu construire une action de formation, un Atelier de médiation numérique (AMN), inspirée du «terrain» et des pratiques actuelles de médiation, augmentée d'une dimension réflexive. Elle s'inscrit dans un dispositif de formation plus large, faisant écho aux pratiques et analyses de l'interface science / technique / société portées par l'institution.

Dans une première partie nous précisons les éléments de contexte, d'orientation des formations, qui permettent de circonstancier et introduire les objectifs de l'AMN, ainsi que les formes pédagogiques développées dans cette formation. La deuxième partie 
resitue les inspirations, sources et ressources méthodologiques qui ont permis d'élaborer le scénario de l'AMN. Nous mettons en exergue les différents éléments du dispositif organisés de manière à construire un espace de réflexivité pour les étudiants, ce qui autorise à détailler davantage tous les objectifs de l'AMN.

Deux sessions de mise en place de l'AMN nous ont permis d'observer l'atelier en train de se faire : dans la troisième partie nous confrontons les stratégies pédagogiques initiales avec nos observations des étudiants en situation, dans une analyse ex post. Nous pourrons alors préciser les fonctions et places prises dans l'AMN par les deux ingrédients incorporés dans le scénario: le prototype et le dispositif d'édition collaborative intégré. Nous présentons un exemple de réalisation dans la quatrième partie, ouvrant sur quelques aspects émergents et inattendus dans les productions des étudiants. Nous concluons ensuite par la comparaison raisonnée avec les autres dispositifs présentés dans ce dossier qui permettent d'approfondir la réflexion sur l'AMN, de penser le processus pédagogique pour nourrir le dispositif et de proposer des pistes d'amplification.

\section{Eléments de contexte : une formation du CNAM à la médiation des sciences et techniques en société}

\section{1. Public en formation}

Les formations dans lesquelles s'insère l'Atelier de médiation numérique (AMN) sont à visée professionnalisante dans le champ de la médiation culturelle des sciences et techniques en société. Elles combinent plusieurs niveaux de formation, pour des publics et des projets professionnels variés, destinés à des métiers proches mais distincts : des métiers liés à l'opérationnel, au terrain (médiation face au public, gestion de projets, mise en place concrète d'opérations, etc.) ; et pour le plus haut niveau de diplomation, les métiers relevant davantage de la conception de dispositifs, de missions plus analytiques ou critiques en médiation, nourris d'histoire des sciences et techniques et de "Science and technology studies» (STS) notamment. Nous renvoyons à l'article de Michel Letté dans ce dossier, pour la présentation d'un autre module de formation (utilisant les voies de la culture ordinaire et le dispositif éditorial « La Lucarne »), complémentaire de l'AMN.

Les publics en formation sont très hétérogènes, à tel point qu'il est difficile d'établir des catégories générales pour caractériser le groupe d'étudiant-e-s en formation en $\mathrm{AMN}^{2}$. Pour la moitié d'entre eux environ, ils ont une formation initiale scientifique ou technique : sciences de la matière, sciences de la vie, études d'ingénieur sont les profils récurrents des étudiants : à titre indicatif, ce sont des masteurants, ingénieurs, jusqu'à des post-doctorants envisageant une poursuite de carrière en médiation. Pour l'autre moitié, les formations relèvent des sciences humaines et sociales, des études littéraires (très peu) et de filières déjà tournées vers le monde professionnel (communication, gestion).

6 Ils ont un premier point commun cependant: tous les étudiants ont, au-delà de leur formation initiale, un parcours professionnel et une expérience solides. Parmi ces expériences professionnelles, une large partie concerne la médiation culturelle, en un sens large, recouvrant des activités de communication, animation, journalisme, production audio-visuelle, etc. Avant d'entrer en formation cependant, leur activité n'est pas toujours située à l'interface science / technique / société, et peu d'entre eux 
mobilisent une expertise de type STS. Une minorité a une expérience professionnelle totalement hors du champ de la médiation (on peut estimer à $10-20 \%$ cette proportion, qui change d'une année sur l'autre). Cette structuration correspond aux deux grands types de public présents au CNAM de manière plus générale : d'une part, des adultes qui visent une évolution de leurs pratiques, répondant à l'évolution de leur métier et cherchant une progression dans leur carrière, et d'autre part des adultes qui envisagent une reconversion professionnelle. Il faut ajouter quelques dilettantes, adultes curieux d'acquérir des compétences en médiation, mais sans couplage avec un projet professionnel. Selon leurs ambitions et projets, ces adultes en formation professionnelle continue visent une qualification, qui impose de suivre quelques unités d'enseignement, ou une certification (un «Certificat de compétences» du CNAM ou un diplôme de type Licence Professionnelle). Les publics en formation sont appelés "auditeurs» ou "étudiants ", dans le jargon de l'institution, qui n'accueille en réalité pas de public avec un statut d'étudiant. Nous n'utiliserons que le terme d' «étudiant» à partir de maintenant.

\section{2. Objectifs pédagogiques}

7 Pour assurer une formation complète à la médiation des sciences et techniques en société, les parcours articulent plusieurs modules, des plus théoriques aux plus pratiques. Un versant analytique et critique de la formation vise à acculturer les étudiants aux problématiques de l'interface science / technique / société, à les nourrir de réflexions issues du champ des «Études de sciences " (ou « Science and technology studies ») et à mobiliser ces outils pour l'analyse de controverses socio-techniques actuelles.

Côté pratique, les modules offrent des séquences de formation à la production écrite (type journalistique), orale (en médiation humaine, en radio), audio-visuelle (production de film documentaire, par exemple), et l'AMN est axé sur la mobilisation des outils numériques pour la médiation. Le tout constitue une formation à la fois pratique et critique, passant par la pratique et la familiarisation avec les outils standard : il s'agit de mettre les étudiants (professionnels et futurs professionnels) en capacité d'analyser euxmêmes les enjeux des dispositifs qu'ils mobilisent, et de les faire progresser en cherchant à dépasser les cadres habituels de la médiation culturelle, en innovant. Nous renvoyons le lecteur à l'article de Michel Letté dans ce volume présentant une autre modalité pédagogique, complémentaire de la nôtre, reposant sur une heuristique de la «culture ordinaire des sciences et techniques en société » et sur le dispositif «La Lucarne » (un Atelier collaboratif), lesquels permettent de mobiliser et développer les capacités d'analyse critique des médiateurs en formation.

Dans cette logique, l'AMN intègre les facettes multiples des métiers afférant à la médiation, en articulant une production effective (prototype de dispositif) avec une appropriation de la démarche de conception de ces dispositifs, et une capacité à la réflexivité : ces trois dimensions fonctionnent de manière cohérente au sein de l'AMN et la construction de l'articulation entre elles est précisément au centre de notre réflexion pédagogique.

10 Pour saisir tous les objectifs de l'AMN il est nécessaire d'en présenter maintenant les sources d'inspiration d'une part, et la structure, l'organisation, les enjeux d'autre part. 


\section{De Museomix à l'Atelier de médiation numérique et ses enjeux}

11 Pour élaborer l'AMN nous avons puisé dans les nombreuses initiatives de médiation, de nature «numérique », et qui s'inscrivent dans un contexte de renouvellement actuel des pratiques de médiation (III. 1). L'inspiration première pour concevoir l'AMN a été tirée d'une action en particulier, le dispositif Museomix, dont nous présentons les grandes lignes (III. 2) avant de montrer la structure effective de l'AMN (III. 3) et clarifier les enjeux de ce dispositif (III. 4).

\section{1. Le renouvellement récent de la médiation des sciences et techniques en société}

12 Depuis les développements de la "vulgarisation» des sciences et techniques au XIX siècle, jusqu'aux politiques plus récentes en faveur de la «culture scientifique et technique ", les pratiques de médiation des STS ont beaucoup évolué (Bensaude-Vincent, 2013). Depuis les années 1980, ce sont les efforts conjugués des politiques publiques et des activités associatives qui ont contribué à redéfinir les cadres d'intervention en la matière. Les évolutions des pratiques de médiation des STS auxquelles répondent l'AMN peuvent être saisies à travers deux initiatives, inscrites dans cette histoire, et très actuelles: le projet InMediats et les enquêtes produites au sein de l'Ecole de la médiation.

InMédiats (acronyme de "Innovation - Médiation - Territoires ») ${ }^{3}$ est un projet inscrit au Plan d'investissement d'avenir (PIA) se posant comme un laboratoire de développement de pratiques innovantes en médiation, d'exploration de pratiques, d'élaboration de prototypes. Il fonctionne pour nous comme un indicateur de tendances, qui permet de faire évoluer les contenus de l'AMN, sans pour autant les déterminer. On souligne ici, notamment, le fort impact du Mooc piloté par InMédiats, qui a été un instrument de diffusion de pratiques innovantes.

14 L'Ecole de la médiation ${ }^{4}$ de son côté a mené une réflexion sur les compétences mobilisées par les médiateurs aujourd'hui, en les répertoriant et cartographiant, soulignant entre autres l'importance de «S'appuyer sur les innovations numériques et technologiques » et de « proposer des formes participatives » de médiation.

InMediats et l'Ecole de la Médiation sont deux opérations elles-mêmes inscrites dans des cadres mariant associatif et institutionnel, ce qui fait leur intérêt et leurs limites. L'Ecole de la Médiation, par exemple, à travers son enquête a tendance à figer les compétences décrites, ce qui poussera à terme à transformer cette cartographie en référentiel de compétences plus ou moins partagé par les opérateurs de la médiation des STS. L'AMN essaie, dans la mesure de ses moyens et ambitions, de ne pas cantonner la formation à ces strictes définitions de compétences.

Ces tendances et évolutions remontent aux débuts des années 2000, à une échelle plus globale et concernent les transformations des formations en médiation culturelle, sous l'impulsion du numérique. Les projets comme CampusCultura ont été à la fois initiateurs, porteurs de nouvelles promesses de formation (numériques et à distance), et ils ont été analysés dans les travaux réalisés avec et autour d'E. Fichez ${ }^{5}$. 


\section{2. Museomix et le design thinking}

17 Au-delà des laboratoires InMédiats et Ecole de la médiation, l'AMN puise dans les ateliers collaboratifs Museomix ${ }^{6}$ et la démarche de design thinking sur laquelle ils reposent. Un Museomix est un rassemblement organisé dans un Musée souhaitant faire évoluer ses pratiques : après une préparation de 6 mois environ, il s'agit, sur 3 jours, de réunir des médiateurs, conservateurs, personnels du Musée, d'une part, et une communauté de " hackers », designers, programmeurs, architectes, scénographes, etc. d'autre part.

En suscitant des projets collaboratifs, en temps limité, il s'agit de «remixer» les collections d'un Musée, d'expérimenter des nouveaux usages possibles, de prototyper des nouveaux dispositifs de médiation et d'appropriation du Musée. En cela, un Museomix s'apparente à un «Hackaton $»^{7}$ de Musée et, dans sa mise en œuvre, il mobilise un «Laboratoire de fabrication » (FabLab $\left.{ }^{8}\right)$ ad hoc.

19 La démarche Museomix repose avant tout sur une communauté, ouverte et en renouvellement permanent ${ }^{9}$. Elle est à la fois locale, car des communautés existent à l'échelle de villes ou régions. Et globale car l'ensemble de ces communautés forment en soi une grande communauté de partage et de collaboration, mobilisable sur des rassemblements programmés avec une structure culturelle: initialement des Musées, mais les contextes se sont élargis et internationalisés, avec des scènes de spectacles, galeries, théâtres, etc.

Museomix offre un mode de génération de dispositif innovant, orienté sur la médiation (même si, somme toute, la médiation n'est qu'un aspect du dispositif). Cela nous fournit un modèle de dispositif d'action de médiation, que nous avons adapté dans un cadre pédagogique sous forme d'AMN. En toile de fond méthodologique de ces «remixages » se trouve la démarche de design thinking (Brown, 2014). L'approche est inspirée des méthodes des designers qui visent à concevoir un objet ou un dispositif pour lequel l'expérience de l'utilisateur est primordiale: ne pas imposer l'objet, ne pas répondre strictement à un besoin préconçu, mais le rendre « désirable », faciliter et développer son utilisation. La démarche peut se décomposer en quelques étapes clés : ce sont autant de principes qui, dans l'action, ne se suivent pas strictement linéairement mais sont itérés de manière répétée.

21 Dans une démarche de design thinking, la première étape consiste en une enquête de type ethnographique, sur le terrain, pour observer les comportements et comprendre les expériences des utilisateurs (en contexte muséal, pour Museomix, mais dans n'importe quel terrain pour les designers). Ensuite, seconde étape, il s'agit de définir une problématique de conception et de la circonscrire. Puis de générer dans la foulée des solutions, de manière créative, ouverte, jusqu'à élaboration de concepts adéquats à la problématique. Les étapes suivantes visent à expérimenter, sur la base d'un prototype, pour déterminer la viabilité des concepts et tester le dispositif sur des utilisateurs. Cette démarche de conception incorpore une forte dimension collaborative et interdisciplinaire d'une part, et procède de manière itérative à partir de l'étape de prototypage, d'autre part. Museomix rassemble sur son site une grande série de prototypes réalisés au cours des opérations, dans différents lieux ${ }^{10}$. 


\section{3. Structure et temporalité de l'AMN}

22 L'AMN détourne (ou adapte) les principes de Museomix et la démarche de design thinking, pour répondre à un scénario pédagogique qui se développe sur les bases suivantes :

- Mise en place de groupes de travail de 4 étudiants (idéalement), pour faciliter la collaboration en situation de formation ;

- Elaborer un calendrier de conception, étalé sur 5 mois (contrairement aux 3 jours d'un Museomix) ;

- Choisir un lieu et des usages à explorer : étant au CNAM, les étudiants travaillent dans les collections permanentes du Musée des arts et métiers. Ils sont confrontés à des objets (de patrimoine) et des usages institués (proposés par les services du Musée) dans un lieu muséographié ;

- Le FabLab sur lequel s'adosse un Museomix n'est pas transposable en l'état.

étudiants ont à leur disposition une "Boîte à outils numériques", dans laquelle ils puisent des ressources, à compléter en fonction de leurs projets. L'autonomie d'exploration des outils numériques existants est impérative, et n'est pas perçue comme un obstacle pédagogique. Au contraire, la découverte de nouveaux outils vient parfois nourrir la "Boîte à outils " partagée, sans cesse mise à jour (étant donné le foisonnement d'outils ouverts, libres et gratuits générés aujourd'hui).

Le processus se déroule sur une temporalité assez longue, scandée par trois regroupements des groupes d'étudiants (Ateliers en classe) pour cadrer la méthodologie et définir les enjeux de chaque étape du design des dispositifs. Chaque Atelier lance une étape, mais c'est aussi un moment de construction de la réflexivité : or, étant donné la temporalité très courte d'un évènement Museomix, la réflexivité est difficilement concomitante de l'évènement lui-même. Dans Museomix le temps de l'action est plutôt séparé du temps de la réflexion sur les pratiques, qui peuvent se développer après l'évènement, en capitalisant sur l'expérience de l'évènement, mais sans rejouer la démarche de conception une seconde fois. L'AMN couple la conception avec une dimension réflexive, en quelque sorte de manière synchrone. En outre, chaque Atelier de l'AMN fait intervenir des professionnels différents apportant leur expertise et encadrant de fait la production de ces Ateliers.

Le premier Atelier présente les pratiques de médiation du Musée des arts et métiers (pratiques de l'institution) et en contre-point entame le processus d'enquête sur le terrain, visant à diagnostiquer et documenter les pratiques actuelles (et non pas seulement les pratiques souhaitées par l'établissement). Il fixe le cadre méthodologique et délivre les rudiments de l'enquête ethnographique, au départ du processus de 5 mois. La première phase d'enquête est lancée, elle se poursuit en autonomie par groupe avec un suivi sous forme de tutorat, par un dispositif que nous allons préciser. C'est dans cette phase que se joue également la première approche des objets de collections du Musée : l'enquête combine donc plusieurs regards et mobilise une expertise de type STS à la fois orientée sur la problématique de muséographie / médiation et pour donner sens aux objets patrimoniaux eux-mêmes au travers d'éléments d'histoire des sciences et techniques. Elle se termine avec le deuxième Atelier qui assure un bilan, une synthèse des observations par groupe. 
30 Le deuxième Atelier, organisé 2 mois environ après le premier, est le point de transition entre l'observation et l'action : brainstorming, premières pistes de travail, mobilisation de la «Boîte à outils numériques" constituent l'essentiel de ce temps d'action, en temps limité et en classe. Il s'agit de poser les bases du processus créatif, amplifié par la suite dans chaque groupe: prototypage, premières réalisations et tests "grandeur nature " dans la mesure du possible, en autonomie et suivi par l'équipe encadrante.

31 L'Atelier 3 conclut le processus : les résultats des prototypes et tests, mais surtout la démarche globale sont soumis à des professionnels pour faire le bilan, asseoir les acquis, pointer les écueils de chaque démarche.

32 Les intervenants et interventions sont de nature différente à chaque étape : médiateurs et enquêteurs pour le premier Atelier ; designers et facilitateurs d'innovation pour le deuxième ; professionnels de la communication, de la médiation, responsables de structures de médiation pour le dernier.

33 Les premiers AMN ont été lancés, sur l'année universitaire 2014-15, sur une démarche très empirique et en ajustant les méthodologies au fil de l'année. Sur l'année 2015-16, la démarche a pu bénéficier de la première année expérimentale en proposant un cheminement plus cadré, assurant le suivi de 6 groupes de 4-5 étudiants (jauge similaire pour les deux années de l'AMN).

\section{4. Les objectifs et enjeux de l'AMN}

Nous pouvons maintenant préciser les objectifs de l'AMN de manière plus exhaustive. Reposant sur une démarche inspirée du design thinking et s'appuyant sur les expériences de Museomix, l'AMN vise plusieurs objectifs : certains sont intégrés dans ces démarches, d'autres objectifs dépassent ce qui est produit dans ces processus. Le premier objectif, c'est celui de créer un dispositif de médiation numérique, dont le prototype est la colonne vertébrale. Mais l'AMN ne se limite pas à concevoir un dispositif, l'ambition est autre : on pourrait presque dire que le prototypage est un prétexte pour atteindre au moins quatre autres objectifs (sans les hiérarchiser) :

35 - Apprendre à collaborer : la forte interdisciplinarité et l'hétérogénéité des parcours, profils et projets professionnels des étudiants sont à la fois une richesse et un obstacle à la collaboration. La collaboration, nécessaire à l'opération, se construit dans le temps, en trouvant un terrain commun, une culture commune à chaque groupe. Dans un Museomix la collaboration est par ailleurs décisive, d'autant plus que l'évènement est de courte durée ;

36 - Comprendre la démarche de conception et savoir la mobiliser en toute situation : audelà du produit / prototype de l'AMN, c'est bien la démarche de conception qui est transposable dans d'autres situations professionnelles, et qui permettra de générer d'autres dispositifs innovants, quel que soit le contexte. Cette démarche ne peut être comprise et intégrée qu'au prix d'une documentation systématique de leur progression. Les étudiants élaborent un document de travail rassemblant leurs enquêtes, réflexions, brainstorming. Conserver la trace du processus est un facteur clé pour atteindre pleinement cet objectif de l'AMN ;

37 - Élaborer leur dispositif de manière réflexive. Documenter et éditer, c'est enfin, et surtout, la clé pour élaborer de manière réflexive, comprendre " comment » le dispositif est construit et pouvoir généraliser la démarche : d'une part, analyser la conception du 
dispositif et ses conditions de possibilité ; d'autre part, réfléchir à la transposabilité de la démarche dans d'autres contextes et conditions. Le document sert tout au long du processus pour le groupe, il est indispensable pour la relecture finale de la démarche dans laquelle les étudiants sont entrés, et pour construire les dialogues avec les différents experts associés aux différentes étapes de l'AMN ;

- Construire des compétences dans le domaine STS et en histoire des sciences et techniques: même si cet aspect constitue un second plan dans le processus, il est indissociable des compétences liées à la médiation des sciences et techniques, qui est ellemême devenue une problématique abordée par les STS. Dès lors que les objets patrimoniaux et historiques sont au cœur du prototype conçu par les étudiants, ils donnent sens à ces objets en puisant dans l'histoire des sciences et techniques. Cet objectif est congruent aux autres modules de la formation délivrée au CNAM. Par ailleurs, l'expertise dans le domaine est essentiellement portée par L. Petitgirard dans l'AMN, mais selon les dispositifs créés les étudiants peuvent aller eux-mêmes chercher d'autres voix, et doivent apprendre à assurer une médiation supplémentaire de ces savoirs et expertises (voir plus loin dans le texte, un exemple avec le processus nommé « EC2 »).

\section{Le processus pédagogique : stratégie pédagogique et premiers retours d'expérience}

Pour atteindre ces objectifs nombreux, nous avons élaboré un processus pédagogique, partant de la structure globale et la temporalité de l'AMN présentées ci-dessus, en intégrant un dispositif central d'édition collaborative («EC1» dans la suite) facilitant la collaboration et l'appropriation de la démarche au sein de chaque groupe en formation (IV. 1). L'intégration de l'EC1 et son rapport à la construction du prototype est partie intégrante d'une stratégie visant à mettre les étudiants en situation de création puis de réflexion (IV. 2). Dans cette partie nous discutons ces choix, et les confrontons aux premières observations sur l'AMN en « train de se faire » (IV. 3 et IV. 4).

\section{1. Un dispositif d'édition collaborative pour faciliter la collaboration et l'appropriation}

40 Le passage par la pratique de conception est indispensable pour une appropriation de la démarche, mais sans un outillage documentaire et éditorial, l'AMN resterait un simple exercice de conception. Nous avons donc ajouté au scénario pédagogique un système d'édition qui permet (et facilite) le travail de documentation, d'édition et de collaboration recherché. Pour l'enseignant il est décisif pour lire l'avancement, saisir les difficultés de chaque groupe, accompagner le processus d'élaboration et sert de document final pour l'évaluation du travail du groupe. Les étudiants sont ainsi suivis et accompagnés dans toutes les dimensions du processus de recherche: ethnographique, muséographique, médiatique et historique. Et c'est dans cette dimension éditoriale collective que se trouve l'espace de réflexivité en construction.

41 Le processus EC1 (édition collaborative 1) est pensé pour se dérouler en continu, depuis le premier Atelier jusqu'à la conclusion. Les étudiants n'étant pas habitués à manier ces outils d'édition, EC1 doit reposer sur des systèmes très simples (pour ne pas dire rudimentaires) d'édition collaborative, tel qu'on les trouve en ligne : framapad, hackpad 
pour les plus usités - libre à chaque groupe de décider d'utiliser d'autres outils plus élaborés s'ils le souhaitent. L'accès doit être immédiat, l'utilisation ne doit pas demander de maîtrise technique numérique sous peine de dissuader son utilisation. L'appropriation du système par les étudiants est conditionnée à cette simplicité d'usage.

\section{2. Stratégie pédagogique : créer pour réfléchir}

Le choix de détourner une action de type Museomix est très directement lié à une stratégie pédagogique qui place l'acte de création au cœur du processus pédagogique en l'articulant avec le processus réflexif. Cette présentation sert également d'écho pour l'analyse produite par A. Bernard et L. Petitgirard dans ce dossier, confrontant des stratégies " créer pour réfléchir » comparables mais insérées dans différents dispositifs de formation et articulant ces stratégies à celles d'autres dispositifs misant plutôt sur son complémentaire : « réfléchir pour créer ».

Dans Musoemix, il s'agit de créer et proposer des dispositifs de médiation, en rupture avec les pratiques du lieu. Dans l'AMN il faut élargir ces objectifs propres à Museomix au profit de la mise en œuvre du processus réflexif. Dans une visée pédagogique, nous adaptons les processus et les objectifs de Museomix, en focalisant sur l'acte de médiation, sur le médiateur et l'élaboration de sa capacité réflexive.

Chronologiquement, l'enquête ethnographique initialise l'AMN, et en ce sens la réflexion sur la médiation débute avant la création: cependant, et pour clarifier la stratégie, le processus critique et réflexif qui nous intéresse démarre seulement avec (et se poursuit après) la création du prototype (qui est évidemment nourri des conclusions de l'enquête). Ce processus est de nature très différente de l'analyse observante des usages de médiation institués. Il ne s'agit plus d'observer mais de créer un dispositif de médiation et ses usages. Le but est donc de promouvoir des usages (existants mais peu courants ou refreinés), anticiper des usages émergents, imaginer des usages nouveaux et en rupture avec l'existant, puis les confronter au public. C'est sur la base du prototype créé que se construit ensuite la mise à distance critique du dispositif, opération rendue possible parce qu'il est fabriqué et maîtrisé, contrairement aux collections muséographiées sur lesquelles les groupes n'ont pas de prise. Leur prototype est maitrisé dans ses intentions, dans les usages autorisés (et même parfois prescrits), dans les contenus diffusés. L'AMN place donc les (futurs) médiateurs en situation de construction pour réfléchir sur leur création.

Cependant, le processus n'est pas unidirectionnel: réfléchir permet de recréer, d'améliorer, de réorienter, de corriger... dans un processus qui est itératif entre réfléchir et créer. C'est une transposition des itérations propres à la démarche de design thinking, en un sens, mais la transposition ne peut pas être directe, encore une fois, car ce n'est pas le résultat en tant que tel (prototype et ses dérivés) qui nous intéresse, mais la démarche et le processus réflexif associé : c'est là où intervient $\mathrm{EC} 1$, comme espace ouvert pour la réflexivité.

\section{3. Le prototype et le dispositif EC1 - retour d'expérience}

Nous avons choisi délibérément de faire de l'étape de prototypage une étape clé. A l'expérience le prototype s'est clairement imposé comme central dans le processus. 
L'observation empirique a également révélé plusieurs effets émergents liés aux efforts de conception du prototype, autorisant une analyse ex-post plus complète du processus.

En premier lieu, le prototype permet d'introduire le processus de réflexion, ce qui était bien l'intention de l'AMN, c'est ainsi qu'il a été présenté et abordé pédagogiquement. Il est central dans le processus dialogique avec les «extérieurs »: autres étudiants, enseignants, professionnels, experts qui entrent en discussion sur les projets, en critiquant en première intention le prototype. Le prototype est un point focal pour le processus dialogique. De manière générale, le prototype devient le support "matériel » de la réflexion: on fait évoluer le prototype dans ses attributs, ses fonctions, au fur et à mesure de la réflexion. Le groupe agit à travers le prototype et sur le prototype.

Le prototype intègre une partie des résultats de l'enquête préliminaire, ce qui est souhaité par construction, mais pas toujours acquis, ni évident pour les groupes.La relation prototype-enquête est ensuite un processus fait d'aller-retours, dans lequel le prototype devient un prisme : au fil de l'évolution du prototype le groupe peut relire et réinterroger le lieu, les collections et leurs fonctions à travers leur propre production. Or dans la conception initiale de l'AMN, l'étape d'enquête était close à partir du moment où l'étape de prototypage s'engage. L'enquête est consignée, éditée, sous forme de document partagée, dans ECI. A la lumière de l'expérience, l'enquête s'avère ne pas être seulement un document figé à l'étape du prototypage, elle est en fait relue, revue, parfois reprise sur certains points, à la lumière du prototype.

En outre, le prototype a un effet intégrateur des compétences "périphériques" de chaque étudiant, c'est-à-dire des compétences qui n'avaient pas été pensées comme centrales dans leur projet de formation initiale, liées à leur expérience professionnelle. Ces compétences sont utiles au déploiement du prototype, que chaque groupe peut insérer dans le prototype (compétence en programmation, en design, en graphisme, etc.). C'est un effet de fertilisation croisée des compétences, figure classique de la formation d'adultes dans un contexte tel que le CNAM ${ }^{11}$.

Enfin, et c'est à la fois un obstacle et un avantage qui avaient été sous-estimé dans la mise en place des premiers AMN : le prototype a un effet de désacralisation. Le prototype introduit d'emblée un nouvel usage dans un lieu qui était perçu comme "sacré » et immuable. La révérence des étudiants vis-à-vis des collections patrimoniales et du lieu avait pu nous surprendre, car elle semblait interdire une réflexion sur les usages, et représentait un obstacle dans le processus de construction du prototype en restreignant l'horizon des possibles aux frontières du «correct dans une institution muséale » (voir plus loin, dans la présentation du projet "Voyage dans le temps»). Cet obstacle peut handicaper considérablement la création s'il n'est pas franchi rapidement. Mais l'obstacle peut être levé, et en tout cas plus facilement franchi, dès la première matérialisation d'un prototype (qu'il soit finalisé ou abandonné ensuite).

51 Des observations du processus en acte, il apparaît assez clair que le prototype revêt une grande importance au fil du processus pédagogique, alors qu'il a été pensé, initialement, dans le processus pédagogique comme un "prétexte ", comme un support pour initier la réflexion. Malgré tout, au final il n'a qu'une importance relative puisque c'est la démarche traversant l'AMN qui importe. Il est une sorte d'échafaudage nécessaire, particulièrement choyé par les étudiants qui organisent et construisent "leur " prototype. La réussite, l'esthétique, la mise en fonction parfaite du prototype comptent à leurs yeux, malgré tout, et même si cela n'entre pas en ligne de compte des évaluations... 
qui portent beaucoup plus sur les contenus de l'EC1. Pour analyser complètement le processus, il est nécessaire de faire le point sur la place prise par EC1, et son articulation avec le prototype.

\section{4. Quelles sont les fonctions d'EC1 et comment s'articule-t-il au prototype?} enseignants / encadrants, entre étudiants et professionnels, et entre groupes d'étudiants qui peuvent suivre les progressions des uns et des autres : les éléments consignés sont mobilisables dans les processus dialogiques assurant l'analyse critique des objets créés (prototype), de la démarche dans son ensemble, et la construction de l'expertise des étudiants (en matière de médiation, de STS et d'histoire des sciences et techniques, en particulier).

En observant le déroulement de l'AMN, on peut décrire les fonctions réalisées effectivement par EC1 et caractériser son positionnement dans le processus pédagogique.

En premier lieu, EC1 fait très clairement ressortir les difficultés, les interrogations et les obstacles rencontrés par chaque groupe, qui écrivent de manière très simple, très directe, l'ensemble des problématiques de leur projet. EC1 est approprié par tous les étudiants, mais pas utilisé de manière uniforme : certains groupes conservent un format de texte plutôt «brut ", linéaire, avec peu d'éditorialisation ; d'autres travaillent à la mise en forme et synthèses régulières sur les échanges. Les moments d'Atelier en classe sont les temps propices à ces efforts d'éditorialisation. EC1 est en tout cas le support d'échange privilégié et s'impose comme système de facilitation de la collaboration.

Deuxièmement, EC1 entre en interférence avec le processus de conception: l'étape d'enquête génère une documentation très riche, mais une fois entré dans le prototypage, l'action l'emporte sur l'édition, donc au détriment de EC1. Imaginer, tester, expérimenter, le processus est pris très au sérieux dans l'AMN, comme dans Museomix : en temps limité, Museomix n'offre pas le temps de la réflexivité simultanée, ce pourquoi l'AMN a été pensé sur du temps long. Mais le temps mis à disposition n'est pas la garantie de la construction d'un espace de réflexivité dans l'EC1, ni de son succès. La relation EC1 - prototype est donc problématique.

Pour que le concret de la réalisation ne l'emporte pas sur le temps d'écriture et d'éditorialisation, la contrainte pédagogique (exercée lors de l'Atelier 2 de l'AMN) est décisive, et le suivi entre Ateliers en classe doit réactiver cette contrainte, au besoin: documenter la démarche, pour que EC1 soit maintenu comme espace intermédiaire, espace de collaboration, espace de réflexivité. S'il est piloté correctement, EC1 est un incubateur pour les éléments du prototype et pour le travail réflexif.

Enfin, EC1 sert de "point de référence » aux échanges qui s'opèrent entre étudiants et Plusieurs ingrédients sont nécessaires pour faire d'EC1 l'espace de base pour développer une mise à distance critique : temporalité longue, outil numérique très facile d'accès, consigne pédagogique pour contraindre l'appropriation, mise en place la plus rapide possible d'un mode de collaboration entre étudiants. La collaboration est décisive pour le processus d'écriture et plus fondamentalement pour le processus réflexif qui est à la fois collectif et individuel. 


\section{Exemple du dispositif « Voyage dans le temps »}

Dans l'espace du Musée consacré aux collections d'appareils photographiques, un groupe d'étudiants a proposé un dispositif intitulé « Voyage dans le temps ».

\section{1. Le projet de médiation}

De leur enquête de terrain, au Musée, étaient ressorties plusieurs observations générales et certains usages sont apparus entravés par le dispositif muséographique existant : la muséographie crée en effet un rapport très distancié à l'objet (mis à distance derrière des vitrines), qui devient inaccessible en termes matériel, sensoriel et d'une certaine manière, en termes cognitifs ${ }^{12}$. Ce qui entre en contradiction avec le ressenti des visiteurs de la collection, qui ont un rapport souvent beaucoup plus direct, émotionnel avec les appareils photographiques et surtout une expérience d'utilisation (passée) de ces appareils. La problématique de médiation du « Voyage dans le temps » se construit sur cette contradiction entre expérience de visite et expérience personnelle de l'usager. Le projet vise à résoudre cette "frustration" d'usagers curieux, nostalgiques pour beaucoup : en créant une relation entre ces deux expériences, en réalisant un dispositif de médiation culturelle numérique, le groupe veut proposer une nouvelle expérience de visite, inédite.

Le dispositif «Voyage dans le temps" articule plusieurs modules, dont une "frise chronologique " numérique qui sert à l'exploration de l'histoire des appareils, et un système de jeu dont l'objectif est d'offrir une contextualisation des appareils présentés ${ }^{13}$. La frise intègre les appareils exposés au Musée parmi de nombreux autres contenus: objets non exposés, images photographiques, films, bref tout document donnant à voir une histoire plus complète de la photographie. Tout ce qui n'apparait pas aux yeux d'un visiteur du Musée est rendu accessible par cette «augmentation » numérique (utilisable sur des systèmes portables: ordinateurs, smartphones, tablettes, dès lors qu'ils sont connectés à Internet). Le système est en outre potentiellement accessible à la maison, offrant une visite hors les murs du Musée.

61 Le système de jeu de piste cherche à modifier l'expérience du visiteur d'une part et à faciliter la réinsertion des appareils dans leur contexte historique, économique, culturel et industriel, d'autre part. La première proposition d'interaction (au travers d'une plateforme connectée à Internet) se fait par la saisie d'une année, suggérant que le visiteur choisisse une date marquante: première utilisation d'un appareil photo (ou année de naissance, année symbolique à titre personnel). A partir de cette date, le jeu renvoi d'une part le visiteur à la recherche d'autres objets des collections du Musée, contemporains et susceptibles d'éclairer le contexte précis lié à cette date ; d'autre part, remobilise les données de la frise numérique pour compléter les informations, assez pauvres, mises à disposition in situ sur les collections d'appareils.

\section{2. Les éditions collaboratives dans le projet « Voyage dans le temps"}

Lié à ce projet, le travail EC1 rassemble les enquêtes, échanges et questionnements du groupe, la manière de collaborer et de répartir les tâches (nombreuses, vue l'ambition du 
projet). EC1 dans ce groupe contient aussi des synthèses, des points où se concentrent la réflexion approfondie sur les modalités des médiations déjà en place et celles à créer. De cette phase d'incubation lisible à travers EC1, il ressort progressivement le choix de construire un autre dispositif (qualifié ici d'EC2), perçu comme une nécessité face à la grande quantité de contenus à éditer concernant les appareils, leur histoire, leur contexte de création et d'utilisation. Or les compétences et connaissances du groupe ne suffisent pas pour construire ces contenus, qui doivent par ailleurs être calibrés, édités, pour le dispositif. De manière générale, l'accompagnement sur les aspects d'histoire des sciences et techniques repose essentiellement sur un enseignant (L. Petitgirard) : il est donc nécessairement limité et plutôt d'ordre méthodologique. Dans le cas présent, le groupe a élaboré un dispositif facilitant l'édition de contenus, comme résultat de plusieurs opérations : collecter les données, les avis, les expertises ; les sélectionner, les trier et procéder à une édition ; les mettre en forme pour les rendre utilisables et accessibles.

L'édition de type $\mathrm{EC} 2$ dépasse largement le processus de type $\mathrm{EC} 1$ en termes d'ambitions. Il implique plusieurs opérateurs : les étudiants eux-mêmes d'une part, et des extérieurs d'autre part, qu'ils soient sollicités pour leur expertise ou qu'ils soient des contributeurs moins identifiés. Parmi les experts à associer, il y a les responsables de ces collections au sein du Musée, et d'autres historiens extérieurs au Musée, spécialistes des techniques photographiques. Les étudiants s'impliquant fortement à la fois dans EC1, amplifié dans EC2 en arrivent à asseoir un socle de connaissances sur l'histoire de la photographie et ses enjeux sociaux: ce n'est pas l'ambition première du processus pédagogique, mais un produit dérivé remarquable.

\section{3. Une édition collaborative émergente - EC2}

Ces premières remarques sur l'EC2 peuvent se généraliser à d'autres projets (souvent moins ambitieux tout de même) qui visent à mobiliser des regards et expertises extérieurs dans le prototype. En effet $\mathrm{EC} 1$ possède ses vertus, mais aussi des limites, la principale étant le caractère fermé du dispositif: l'édition et la collaboration sont restreintes aux acteurs-étudiants du groupe, il est conçu pour cela, mais il empêche de fait d'associer d'autres contributions, d'autres productions qui pourraient enrichir considérablement l'édition (à une exception près: les enseignants et professionnels encadrant le processus interagissent et entrent en dialogue sur la base de ce document, et participent à le transformer, indirectement). Et c'est de manière plutôt involontaire, sans guidage méthodologique ni contrainte pédagogique, que EC1 a été doublé, dépassé et amplifié par un autre processus éditorial apparu dans certains projets seulement, un EC2 émergeant.

Les «moteurs" d'éditorialisation qui ont pu émerger de l'expérience ne sont liés à aucune consigne et ils ont été intégrés de manières diverses aux réalisations des étudiants, parfois au cœur même du prototype imaginé et donc au cœur de l'opération de médiation. Ne répondant pas à une demande cadrée, chaque processus d'édition EC2 est singulier : ce qui nous intéresse ici ce sont essentiellement les conséquences de cet EC2 sur le travail (commandé) en EC1. En effet, dans EC2 se joue une collaboration étendue entre acteurs du projet et acteurs extérieurs, ce qui nécessite un travail d'intermédiation très complexe, bien au-delà des exigences de l'AMN. La mise en place d'une collaboration, sur ce mode EC2, est bien plus difficile. Elle pose parallèlement la question du rôle des différents acteurs, de leur légitimité également à aborder les différents sujets. Tout 
processus de type EC2 rejailli sur EC1, en amplifiant les questionnements et en activant les collaborations internes.

Cela nous amène assez naturellement à nous interroger sur l'effet levier de ce processus éditorial, sur les manières de généraliser aux autres groupes cette amplification. Il est manifeste qu'EC2 existe comme création spontanée du groupe et repose en grande partie sur une appropriation de fait : brider cette spontanéité, ou la forcer, nous apparaitt malgré tout très contre-productif. A l'heure actuelle, nous réfléchissons à des voies d'incitation à ouvrir de tels espaces, plutôt qu'à des contraintes supplémentaires.

\section{VI. « Hacker » et collaborer : penser le processus pédagogique}

67 À l'image des autres dispositifs discutés dans ce dossier, le processus

pédagogique de l'AMN engage les étudiants dans un travail à la fois individuel et collectif : inciter l'individu à changer de référentiel (un changement de l'être), à opérer un décentrement, propre à tout travail en médiation, et un changement de perception sur les outils numériques, qui font désormais partie de nos quotidiens (VI. 1 et VI. 2). De manière plus diffuse, et inégale selon les voies prises par les groupes, le processus conduit les étudiants à construire leur expertise en STS et histoire des sciences et techniques, appuyée par le travail sur les objets du Musée. A un niveau collectif, il s'agit fondamentalement d'apprendre à collaborer, d'apprendre à concevoir et réfléchir ensemble: sur ce second point nous pourrons entrer en comparaison directe avec l'analyse de M. Guedj concernant les dispositifs autour de l'exposition «Regards croisés sur la lumière » (VI. 3).

\section{1. Se décentrer et désenchanter le «numérique »}

Dans l'AMN, inséré dans une formation plus large au CNAM, le travail réflexif vise à développer la capacité critique sur les dispositifs de médiation, et de manière plus générale sur l'acte de médiation lui-même, sa signification, sa portée, ses enjeux : c'est à ce prix que le processus enclenché est généralisable, transposable dans toute situation de médiation. Ce travail ne peut pas faire l'économie d'une réflexion des étudiants sur le rapport entre l'action de médiation et les outils mobilisés, numériques ici.

Or le numérique bénéficie aujourd'hui d'une aura singulière, une sorte de "magie»: l'adjectif confère un supplément de pouvoir à tout dispositif qualifié de «numérique " (numérique est devenu substantif, mais il est d'abord un adjectif). Or ce n'est pas une panacée, bien au contraire, et le changement à produire chez les étudiants est une sorte de renversement de cette magie, et un désenchantement de la « médiation numérique ».

Dans le processus de l'AMN, il est clair que l'enquête, la première partie, est complètement insuffisante pour créer les conditions d'un changement de référentiel : la seule observation des usages, même la plus fine et la plus acérée, ne suffit pas à prendre conscience des obstacles et problématiques en question. Le changement de référentiel et le décentrement qui s'opère en médiation n'est conscientisé qu'avec l'étape suivante, celle du prototypage, celle de la confrontation à l'outil et sa place dans le dispositif de médiation. Le processus d'édition collaboratif donne la possibilité de confrontation dialoguée, qui donne les moyens de l'analyse et de la critique du processus de médiation 
en jeu, de l'articulation aux outils numériques mobilisés. Le dispositif complet, seulement, assure le renversement de perspective et permet de dépasser les conditions d'une médiation numérique trop " classique » : celle qui correspond à une simple amplification numérique des pratiques de médiation, sans bouleversement de perspective sur les moyens d'utiliser le numérique en médiation. L'EC2 amplifie encore le processus, engageant les étudiants sur des pistes avancées en matière d'édition collaborative, impossibles d'ailleurs sans outils numériques.

72 Soulignons toute l'importance à donner au détournement dans le processus, car c'est l'incitation à détourner, à « hacker ", qui assure la mise en condition pour un changement de perspective sur la médiation et la mobilisation du numérique.

\section{2. Une pédagogie du détournement}

73 Créer pour réfléchir, c'est la voie choisie dans l'AMN, et on pourrait la décliner en « détourner pour créer et réfléchir » pour en préciser une modalité. C'est même, en quelque sorte, une pédagogie du détournement que nous pouvons défendre ici $^{14}$. Le processus de conception et de prototypage dans l'AMN s'appuie en effet sur le détournement d'outils numériques: tous les outils sont acceptables a priori, même (et surtout) ceux qui n'ont pas été conçus pour imaginer des dispositifs de médiation. Il est primordial de laisser libre cours au raisonnement créatif dans la phase de prototypage, donc d'autoriser et d'encourager tous les détournements. L'objectif de produire un dispositif de médiation, répondant à une problématique induite des observations de terrain, donne un cadre à ce processus créatif pour que le détournement ne dérive pas de son objectif.

D'un côté, ce détournement est facilité du fait que les outils numériques ont été programmés pour un usage précis et qu'ils sont en même temps très malléables et ouvrent sur d'autres usages non prévus, non imaginés par les concepteurs même. Facebook et les réseaux sociaux, par exemple, sont des systèmes de communication à destination d'une "communauté ", mais rien n'empêche de dépasser cette fonction de communication (au profit d'un jeu, d'une enquête, etc.). Le couplage de plusieurs outils, les multiples combinaisons possibles, laissent imaginer des prototypes très riches et variés, même si la mise en opération technique peut s'avérer plus complexe que prévue.

D'un autre côté, la dérive n'est pas toujours si facile à éviter. La fascination pour ce potentiel technique d'usages multiples l'emporte parfois sur la problématique de médiation, construite préalablement: les groupes élaborent trop directement leurs prototypes à partir des usages prédéfinis des outils numériques pris dans la «Boîte à outils ", alors qu'il s'agit de faire l'inverse, c'est-à-dire de créer, en les détournant, des usages répondant d'abord et fondamentalement à une problématique pré-alablement identifiée. La focalisation sur l'outil et les usages prédéfinis de ces outils, sont les premiers obstacles qu'il s'agit de contourner. La facilité et le grand nombre d'usages «tout prêts" du numérique autorisent le détournement et sont en même temps le premier « ennemi ».

76 C'est au cours du second Atelier en classe que cette dérive est corrigée, dès qu'elle est identifiée dans la dynamique du groupe. La pédagogie du détournement ne peut pas se passer d'un cadrage très strict et d'un rappel des enjeux, trop facilement mis de côté à ce point précis: repartir de l'usage souhaité, visé ; casser l'idée que les outils sont prédestinés à un usage (celui qui a amené à concevoir et fabriquer l'outil); sortir de l'idée 
qu'il faut copier cet usage. En d'autres termes, il faut se recentrer sur l'objectif de médiation en détournant l'existant des usages numériques. Ce qui signifie se réapproprier les outils et renverser le côté « magique » du numérique : c'est, en soi, une perspective qui dépasse l'AMN et procède d'un changement assez radical de perspective pour beaucoup d'étudiants (volontiers consommateurs d'usages numériques).

\section{3. "Objet frontière ", "Objet intermédiaire » et les modalités de la collaboration}

77 Concernant maintenant la question de l'organisation des aspects collectifs, la place des différents objets créés pour faciliter la collaboration, et le statut de ces objets, il nous parait pertinent de procéder à des analogies avec l'analyse de M. Guedj.

78 Un premier point préalable s'impose quant au processus lié à l'exposition « Regards croisés sur la lumière »: les étudiants ont accès à l'exposition et au document de travail élaboré par les concepteurs de l'exposition. Ce document est qualifié d'« objet frontière » au sens de Star et Griesemer par M. Guedj: il permet d'organiser le travail de mondes sociaux hétérogènes, sans consensus a priori sur les formes à donner à l'exposition et permet d'assurer la négociation et l'évolution du dispositif. Remis entre les mains des étudiants, il sert de base à leur production, de guide de visite autour de l'exposition : le document change de statut, il devient « objet intermédiaire » - au sens de (Vinck, 2009) un objet qui circule dans ce réseau, servant le travail collaboratif pour élaborer leur propre dispositif.

79 Dans le cas de l'AMN, les étudiants n'ont pas de document qui se rapprocherait d'un «objet frontière " qui a servi à la construction de la muséographie (que ce soient les synopsis, scénarii ou avant-projets), ils n'ont accès qu'au dispositif muséographié final. Ils ne peuvent que rarement accéder aux intentions initiales des concepteurs, aux compromis qui ont été faits, et ils jugent avant tout sur le résultat final, in situ, confronté au public. Dans les faits, ils sentent le besoin de reconstruire le fil de ces négociations et compromis (qui pour l'essentiel ont été faits au milieu des années 1990, sauf pour quelques rares espaces rénovés). Leur enquête associe paroles de visiteurs et de responsables de ces espaces au sein du Musée, qui ont gardé la mémoire du processus muséographique. Ils sont donc en situation de reconstruire un document initial avec toutes ces données, ce qui est transcrit dans l'EC1. A l'issue de l'enquête de terrain, EC1 constitue un premier bilan (non sans rapport avec les documents de projet muséographique originels et qui nous évoque l'» objet frontière » des concepteurs de l'exposition chez M. Guedj) et qui devient, à l'issue du second atelier, un «objet intermédiaire " pour les phases de création ultérieure.

Intervient ensuite l'étape de prototypage, introduisant un nouvel objet: le prototype incarne le résultat des négociations du groupe, qui sont par ailleurs explicitées dans EC1. Le statut de ces objets évolue donc, car les étudiants sont en situation de concepteurs. Ils doivent composer avec leurs divergences, dont les sources sont diverses: les cultures individuelles, leurs parcours, ambitions, pratiques et expertises - déjà explicitées plus haut dans leur rapport au Musée et au patrimoine. Les divergences apparaissent très tôt dans le processus de conception, dès lors qu'il s'agit de choisir un espace précis dans le Musée et des objets de collections qui seront investis dans le prototype. Cette question est souvent rapidement résolue, sans quoi il n'est pas possible de concevoir le prototype, mais c'est bien le reflet que des savoirs, des êtres, des appétences pour la virtuosité 
technique ou la sobriété, des mondes sociaux différents sont embarqués dans l'AMN, résultat de l'expérience personnelle et professionnelle de chacun.

Il est donc tentant de se figurer le prototype et $\mathrm{EC} 1$ comme « objets frontières " pour des étudiants placés en situation de conception collaborative. Nous avons décrit plus haut l'articulation entre les deux : c'est pour ainsi dire la combinaison des deux qui devrait constituer un «objet frontière ». Cet « objet frontière » est à la fois modulaire (constitué de plusieurs parties, mobilisables pour les échanges et la négociation), polyvalent et sa "partie EC1» étant écrite, lisible directement par tous, elle a une propriété de standardisation de l'information qui distingue «objet intermédiaire» et «objet frontière » (Vinck, 2009).

Penser en terme d'» objet frontière » rend compte de ces divergences insolubles, qui pourtant doivent converger ponctuellement dans le prototype et s'exprimer dans EC1. Cela renforce le choix d'insister sur la mise en place d'EC1, et sur les moyens de l'amplifier : un « objet frontière » ne s'impose pas a priori, et il faut donner aux groupes toutes les conditions pour qu'il se constitue. Par ailleurs, il est apparu une seule fois au cours de l'AMN que cela ne converge pas : le dissensus l'emportant au sein d'un groupe a résulté dans la scission du groupe en deux sous-groupes. Cela signe en négatif la nécessité d'un tel objet, sans pour autant garantir le succès.

Mobiliser la notion d'» objet frontière » impose des précautions, pour ne pas en faire un usage seulement nominaliste : la combinaison prototype / EC1 permet l'articulation entre opérateurs, facilite les interactions, donne une flexibilité interprétative importante, mais selon les groupes, cette combinaison n'a pas tout à fait la même fonction, et, en particulier, n'a pas nécessairement valeur d'infrastructure de connaissances ${ }^{15}$. C'est dans la mobilisation des éléments d'histoire des sciences et techniques, liée aux objets des collections, que ce trait est peut-être le plus saillant : le travail documentaire, les vertus épistémologiques de ces recherches structurent un ensemble de connaissances partagées, facilitant la convergence des points de vue des étudiants. Nous manquons de recul et de données sur le processus $\mathrm{EC} 2$, mais il y a fort à parier qu'une infrastructure particulière soit observable plus directement dans les modalités de la mobilisation et de l'organisation des divers savoirs et expertises. Lire les processus à l'aune de la notion d'» objet frontière " permet en tout cas de souligner qu'un tel objet est indispensable, mais non suffisant.

\section{4. Conclusion et perspectives pour faire évoluer l'AMN}

L'AMN a été élaboré de manière très empirique, reprenant enquêtes et propositions du terrain de la médiation pour élaborer un module de formation cohérent, complet, ambitieux, qui vise à former des professionnels. Les compétences mobilisées et développées par les étudiants sont très variées, elles correspondent aux évolutions du domaine, quitte à les dépasser (ou les anticiper) sur un certain nombre de points. La combinaison d'une méthodologie de design thinking et d'un dispositif pour assurer une mise à distance critique, simultanée, n'est pas courante.

Introduire dans l'analyse les notions d'» objet frontière » et d' « objet intermédiaire » est notre première tentative de penser le processus plus complètement, certes de manière heuristique, mais néanmoins fructueuse. L'EC1 a été construit « en marchant », il trouve du sens et une assise dès lors que son articulation avec le prototype est clarifiée, et qu'il est intégré dans un « objet frontière » en déploiement. Cet objet est mobilisé de plusieurs 
manières: dans la création, la collaboration, les confrontations dialoguées, et les restitutions finales que nous avons à peine évoquées. Chacune correspond à une professionnalité en construction chez les étudiants : capacité à collaborer, élaborer des médiations de manière réflexive, construire une compétence en histoire des sciences et techniques et dans le domaine STS, en congruence avec les autres modules de la formation délivrée au CNAM. Le moment final de restitution ajoute une dimension de défense du projet, dans sa globalité, des choix initiaux jusqu'à sa concrétisation.

L'AMN, sur la base de la pratique et l'analyse théorique actuelle, peut se déployer sur d'autres terrains: c'est ce que nous envisageons, en suivant d'une certaine manière l'évolution de Museomix dans d'autres lieux et contextes. D'autres musées et centres de médiation s'inscrivent dans un prolongement clair de l'AMN au CNAM. En revanche, une gare ferroviaire, par exemple, s'annonce comme un lieu nécessitant une approche différente : l'expression de l'interface entre technique et société n'est pas muséographiée, néanmoins scénographiée dans un dispositif ouvert, public, complexe et surtout dans lequel les usages a priori sont très différents d'unespace muséal. Ce serait l'occasion de croiser les problématiques rencontrées dans le dispositif de M. Letté, porté sur ces manifestations publiques, ouvertes (la culture ordinaire des sciences et techniques) constituant des médiations « déjà-là » et qui méritent d'être interrogées, et détournées.

\section{BIBLIOGRAPHIE}

Ausubel, D.P., 1968, Educational Psychology: A Cognitive View. New York: Holt, Rinehart et Winston.

Bensaude-Vincent, B., 2013, L'opinion publique et la science. A chacun son ignorance, Paris, La

Découverte, coll. «Sciences humaines et sociales », $226 \mathrm{p}$.

Brown, T., 2014, L'Esprit design. Comment le design thinking change l'entreprise et la stratégie. Montreuil, Pearson Education.

Caspar, P., 2011, La formation des adultes, hier, aujourd'hui, demain, Editions d'Organisation, Paris.

Fichez, E., Benchenna, A., 2005, Le cas CampusCultura : Document de travail. Modèles économiques et enjeux organisationnels des campus numériques, https://hal.archives-ouvertes.fr/hal-01388315 (consulté le 29/10/2017)

Fichez, E., Bal A., 2007, « Préface : L’intégration du numérique dans les formations du supérieur », Études de communication langages, information, médiations, 2007 (Numéro spécial), pp. 7-15.

Fichez, E., 2007, « Campus numériques : des ambitions à l'épreuve des terrains », Distances et savoir, 4(3), pp.299-332.

Las Vergnas, O., 2010, « L'institutionnalisation de la "culture scientifique et technique", un fait social français $(1970-2010) »$, Savoirs, no. 27 (June 5, 2012), pp. 9-60.

Schiele, B., Koster, E. (eds.), 1998, La révolution dans la muséologie des sciences, PUL, Lyon. 
Star, S.L., Griesemer, J., 1989 « Institutional Ecology, "Translations," and Boundary Objects: Amateurs and Professionals in Berkeley's Museum of Vertebrate Zoology, 1907 - 1939 », Social Studies of Science, no. 19 (1989) 387-420.

Trompette, P., Vinck, D., 2009, « Retour sur la notion d'objet-frontière ", Revue d'anthropologie des connaissances $3, \mathrm{n}^{\circ} 1$, no. 1 (22 juin 2009), 5-27.

Vinck, D., 2009, « De l'objet intermédiaire à l'objet-frontière », Revue d'anthropologie des connaissances $3, n^{\circ} 1$, no. 1 (22 juin 2009), 51-72.

\section{NOTES}

1. Voir, pour les dimensions historiques de ces évolutions, l'article (Las Vergnas, 2010). Pour les évolutions plus récentes, on peut suivre les initiatives telles que l'Ecole de la Médiation, évoquée plus loin dans le texte.

2. Pour le dispositif «La Lucarne », adressé au même public, l'hétérogénéité du public n'est pas seulement un élément de contexte, mais un argument pour construire un large corpus de travail pour les étudiants.

3. Voir le site : http://inmediats.fr/

4. Voir le site : http://www.estim-mediation.fr/ L'Ecole est inscrite également au PIA « Égalité des chances et promotion de la culture scientifique ", au sein d'ESTIM - Egalité d'accès aux Sciences et Techniques, à l'Innovation et au Multimédia.

5. Voir particulièrement (Fichez, E., Benchenna, A., 2005) au sujet de CampusCultura ; et les réflexions plus générales sur la problématique de l'intégration du numérique dans l'enseignement supérieur, à travers (Fichez, E, Bal A., 2007) et (Fichez, 2007).

6. Voir le site : http://www.museomix.org/

7. C'est la contraction de «Hacker » et « marathon ». Un " Hackaton » vise à générer de l'innovation sur un mode collaboratif, en temps limité.

8. De la contraction de "Fabrication Laboratory ». Dans le contexte de Museomix, il s'apparente à un atelier mettant à disposition les outils nécessaires aux fabrications durant l'opération. De manière plus générale ce sont des lieux accessibles à tout public, qui réunissent à la fois du matériel, des outils pour la fabrication (notamment des machines pilotées par ordinateur, facilitant la fabrication), et une communauté de «makers», des «bricoleurs » qui assistent un visiteur du FabLab dans son projet de fabrication.

9. Voir la page « Qui sommes-nous? » du site http://www.museomix.org/

10. De manière un peu plus globale, on est tenté d'inscrire la muséographie actuelle du Musée des arts et métiers dans la tradition de la «transmission », une relation linéaire entre l'élément muséologique (dépositaire d'un savoir) et le visiteur (en référence au chapitre 14 « Repenser le Musée à partir de comprendre et apprendre ", dans (Schiele, Koster, 1998)). Malgré les intentions initiales, cette muséographie met de côté, pour l'essentiel, une approche plus constructiviste des savoirs, restreint les possibilités d'interactions, et ne tente pas de « ponts cognitifs » (Ausubel, D.P., 1968)

11. Malheureusement, les dispositifs ne sont pas ouverts et accessibles, certains ont même été tout simplement effacés : il s'agit d'un effet lié aux "droits à l'image » et à l'exposition de matériaux (textes, photos, interviews) liés au Musée des arts et métiers, qui ne peut se permettre de laisser diffuser des «expérimentations » de ce type, perçues comme des distorsions de son image.

12. A titre plus anecdotique par rapport à cette stratégie, rappelons que l'AMN est en lui-même un détournement des processus de conception à l'œuvre dans Museomix. 
13. Ce qui est un point souligné dans (Trompette, P., Vinck, D., 2009), comme étant originel dans la notion de Star et Griesemer, et trop souvent négligé. Dans "Voyage dans le temps ", l'organisation en «frise chronologique » et la classification des appareils photographiques, donnent à cette combinaison un caractère d'infrastructure de savoirs historiques, collectés par le groupe. Ce n'est pas le cas de tous les projets créés en AMN.

14. A titre plus anecdotique par rapport à cette stratégie, rappelons que l'AMN est en lui-même un détournement des processus de conception à l'œuvre dans Museomix.

15. Ce qui est un point souligné dans (Trompette, P., Vinck, D., 2009), comme étant originel dans la notion de Star et Griesemer, et trop souvent négligé. Dans "Voyage dans le temps ", l'organisation en "frise chronologique» et la classification des appareils photographiques, donnent à cette combinaison un caractère d'infrastructure de savoirs historiques, collectés par le groupe. Ce n'est pas le cas de tous les projets créés en AMN.

\section{RÉSUMÉS}

"Hacker" et collaborer: dispositifs pour la formation de médiateurs culturels des sciences et techniques. Ce texte vise à problématiser un processus pédagogique élaboré au CNAM, dans le cadre des formations à la médiation culturelle des sciences et techniques. L'Atelier de médiation numérique (AMN) place les étudiants en situation de conception d'un dispositif de médiation, de manière collaborative et réflexive. Nous présentons les objectifs, modalités et les différentes phases de l'AMN. Nous confrontons la stratégie pédagogique à l'œuvre, qui vise à faire créer (un prototype) pour une meilleure appropriation et mise à distance critique, avec une analyse ex post de l'AMN «en train de se faire ». Et nous analysons le processus à l'aide de la notion d'» objet frontière ", permettant d'entrer en comparaison raisonnée avec d'autres dispositifs de ce dossier.

This text aims at problematizing a pedagogical process developed at CNAM, among cursus in science \& technology communication: Atelier de médiation numérique - AMN (« digital cultural project workshop »). The AMN makes students conceive their own project and prototype, in a collaborative and reflexive manner. We present the aims, modalities and different phases of the workshop. We confront the initial pedagogical strategy, aiming at creating a prototype for a better handling of the process and capacity to think about their creation, with the observation of the AMN in action. We finally analyse the process with the help of the notion of «boundary object ", allowing us to compare it with other processes discussed in this volume.

\section{INDEX}

Keywords : digital mediation, science communication, cultural mediation of science and technology, design thinking, museum of science and technology, boundary objects

Mots-clés : médiation culturelle des sciences et techniques, médiation numérique, design thinking, objet frontière, musée de sciences et techniques 
AUTEUR

LOḮC PETITGIRARD

Maître de conférences, HT2S (CNAM) et labex HASTEC (ANR-10-LABX-0085) 